\title{
Drei Jahre Imatinib adjuvant bei GIST
}

Imatinib hat klar die CML-Therapie vorangebracht. Auch bei anderen Tumorarten profitieren die Patienten von der Therapie mit dem Tyrosinkinasehemmer, etwa Patienten mit gastrointestinalen Stromatumoren (GIST).

Q ei GIST-Patienten konnte jetzt in einer DStudie ein höherer Nutzen einer adjuvanten Langzeittherapie mit Imatinib im Vergleich zur Therapie über nur ein Jahr dokumentiert werden. Das hat die Auswertung der Daten einer internationalen Studie ergeben, an der auch Kliniken in Deutschland beteiligt waren. Jeweils 200 GIST-Patienten erhielten in der Scandinavian-Sarcoma-Group-XVIIIStudie (SSGXVIII) täglich 400 mg Imatinib über einen Zeitraum von zwölf oder 36 Monaten.

Die orale Therapie wurde innerhalb von zwölf Wochen nach der Operation begonnen. Die Patienten hatten GIST, bei denen das Rezidivrisiko hoch war, beurteilt nach

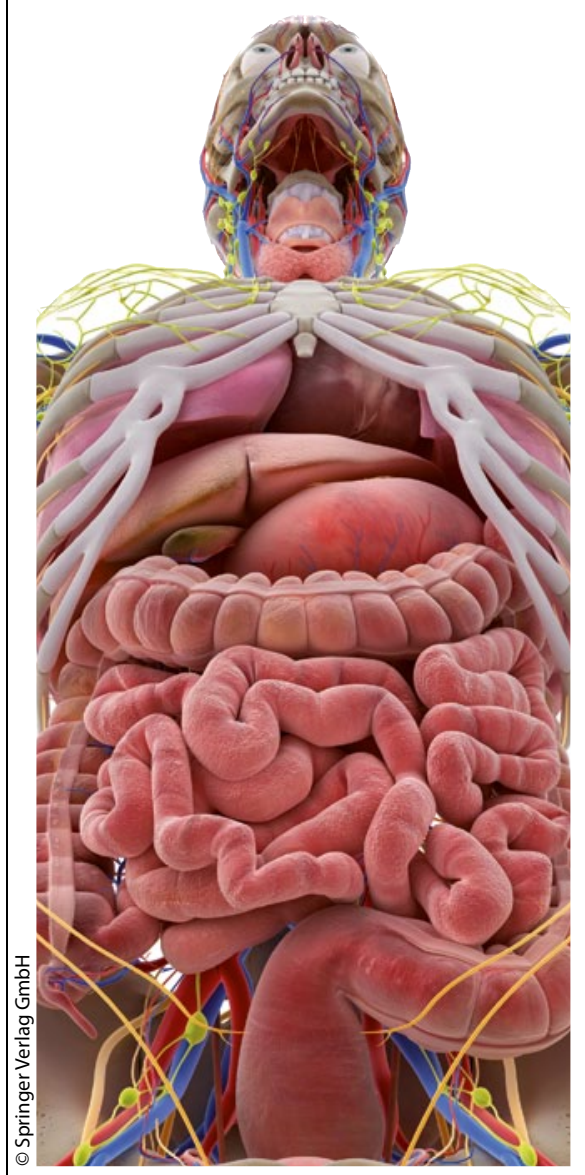

Bei fast jedem zweiten Studienteilnehmer hatte GIST den Magen befallen, bei etwa einem Drittel der Patienten den Dünndarm. den NIH (National Institutes of Health)Konsensuskriterien. Zu diesen Kriterien gehören ein Tumordurchmesser über 5 $\mathrm{cm}$ sowie Tumorrupturen vor oder während der Operation. Patienten mit einem niedrigen oder sehr niedrigen Rezidivrisiko sollten keine adjuvante Behandlung mit Imatinib erhalten.

Im Vergleich zur Therapie über nur ein Jahr war bei Patienten mit einer Behandlung über drei Jahre die Lebensdauer ohne Rezidiv signifikant länger (Hazard Ratio [HR] 0,46). Anders ausgedrückt: Der Anteil der Patienten ohne Rezidiv nach fünf Jahren lag in der Gruppe mit Langzeittherapie bei 65,6\%, in der Vergleichsgruppe dagegen nur bei 47,9\%.

Auch das Gesamtüberleben war bei jenen Patienten, die drei Jahre lang mit Imatinib behandelt wurden, signifikant länger (HR 0,45). Fünf Jahre nach Studienbeginn lebten noch $92 \%$ der Patienten mit Langzeittherapie, in der Vergleichsgruppe mit der kürzeren Therapiedauer dagegen $81,7 \%$ der Patienten.

Die Ergebnisse der Studie haben bereits dazu geführt, dass in Nordamerika, Europa und Asien die Langzeittherapie mit Imatinib über drei Jahre bevorzugt wird. Zu klären bleibt, ob die drei Jahre Therapie künftig ausreichen werden, um tatsächlich das beste Behandlungsergebnis zu erzielen.

Fazit: Der Tyrosinkinasehemmer Imatinib hat neben der Therapie von Patienten mit CML auch die GIST-Behandlung bereichert. Es lohnt sich bei Patienten mit einem hohen Rezidivrisiko offenbar, nach der Resektion das Präparat 36 statt nur zwölf Monate lang zu verabreichen. Ablesen lässt sich der Nutzen sowohl am Parameter „rezidivfreies Überleben“ als auch am Gesamtüberleben. Peter Leiner

Joensuu $\mathrm{H}$ et al. One vs three years of adjuvant imatinib for operable gastrointestinal stromal tumor. JAMA. 2012;307(12):1265-72. - Editorial: Blanke CD. Optimal duration of adjuvant therapy for patients with resected gastrointestinal stromal tumor. Ibidem 1312-3. 